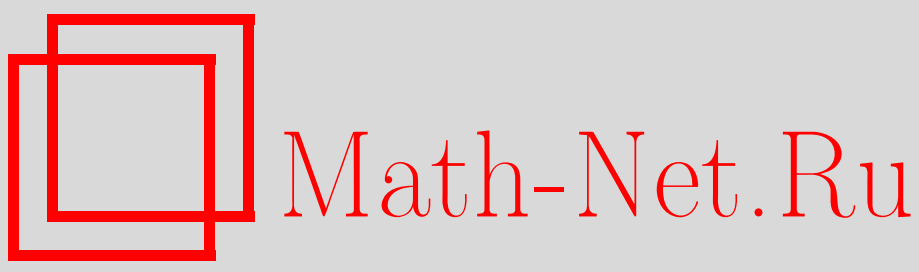

С. В. Тихонов, В. И. Янчевский, Индексы центральных простых алгебр над полями функций проективных пространств над $P_{n, r}$-полями, Матем. сб., 2002, том 193, номер 11, 125-138

DOI: https://doi.org/10.4213/sm694

Использование Общероссийского математического портала Math-Net.Ru подразумевает, что вы прочитали и согласны с пользовательским соглашением http://www . mathnet.ru/rus/agreement

Параметры загрузки:

IP: 34.227 .88 .159

26 апреля 2023 г., 17:35:18 


\title{
Индексы центральных простых алгебр над полями функций проективных пространств над $P_{n, r}$-полями
}

\author{
Пусть $K$ - поле нулевой характеристики, $\mathrm{Br} K$ - его группа Брауэра. В рабо- \\ те получены оценки индексов элементов из $\mathrm{Br} K$ в зависимости от их экспонент \\ для полей функций $K$ проективных пространств и проективных кривых, опреде- \\ ленных над $P_{n, r}$-полем. \\ Библиограффия: 18 названий.
}

\section{§1. Введение}

Пусть $K$-произвольное поле характеристики нуль, $\mathrm{Br} K$ - его группа Брауэра. С каждым элементом $a \in \mathrm{Br} K$ связаны два численных инварианта: его экспонента $e(a)$ и индекс $i(a)$. Хорошо известно, что $e(a)$ является делителем $i(a)$, а классический результат Брауэра утверждает, что $i(a)$ и $e(a)$ имеют одинаковые наборы простых делителей [1], [2]. Важным направлением в теории групп Брауэра является изучение зависимости индекса от экспоненты. К сожалению, никакие общие соотношения (кроме упомянутых) здесь места не имеют, поскольку зависимость между экспонентой и индексом причудливо меняется с изменением $K$. В этой ситуации естественной является конкретизация упоминавшейся выше проблемы.

Пусть задан некоторый класс полей. Найти зависимость межсду әкспонентами и индексами әлементов групп Брауэра полей этого класса.

На этом пути получен ряд важных результатов. Например, известно, что в случае класса полей конечных (или, более того, $C_{1}$-полей) $e(a)=i(a)$ (это классические теоремы Веддербарна и Тзена).

Локальная и глобальная теории полей классов показывают, что предыдущее равенство имеет место и в случае классов локальных или глобальных полей. Совпадение экспоненты и индекса установлено недавно также и в случае полей как повторных, так и двойных формальных степенных рядов с коэффициентами в алгебраически замкнутом поле (см. [3], [4]), как часть обшей гипотезы о совпадении экспоненты и индекса элементов групп Брауэра над $C_{2}$-полями. Однако еще в первой половине прошлого века (Р. Брауэр, [5]) было показано, что экспонента не всегда совпадает с индексом и потому ответ на вопрос об их зависимости не может быть выражен в терминах теоремы о совпадении. С другой стороны, рассмотрение полей рациональных функций от бесконечного числа переменных показывает, что в классе таких полей при фиксированной экспоненте могут сушествовать элементы произвольно большого индекса. Наконец, если рассматривать поля функций $K$ на

Работа выполнена при частичной финансовой поддержке фонда INTAS (грант № 99-817). 
алгебраических многообразиях, определенных над произвольным полем $k$, то тот же эффект неограниченности индексов при фиксированной экспоненте может возникнуть из-за элементов группы Br $k$. Таким образом, если интересоваться проблемой ограниченности индексов при фиксированной экспоненте для класса полей функций на алгебраических многообразиях, являющегося во многих отношениях одним из наиболее важных классов полей, то естественно в качестве полей определения таких многообразий рассматривать поля $k$, в которых соответствующая проблема ограниченности индексов имеет положительное решение. Ярким примером плодотворности такого подхода является недавний результат Д. Солтмэна [6], [7], показавшего, что если $K$ - поле функций на гладкой проективной кривой с полем $p$-адических чисел в качестве поля определения, то для любого натурального $n$ взаимно простого с $p$ при $e(a)=n$ индекс $i(a)$ является делителем $n^{2}$. Причем эта оценка не может быть усилена в том смысле, что существуют алгебры экспоненты $n$ и индекса $n^{2}$.

Все же, если поле $k$ достаточно произвольно, трудно ожидать получения оценок такого точного типа. До недавнего времени одним из основных методов получения оценок индексов в общей ситуации являлся метод “контролируемого” расширения скаляров, убивающего ветвление с последующей оценкой индексов неразветвленных элементов. На этом последнем этапе важное значение имеет результат М. ван ден Берга [8], дающий оценку индексов для неразветвленных элементов.

В данной статье мы рассматриваем класс чисто трансцендентных расширений $K$ конечной размерности над так называемыми $P_{n, r}$-полями и показываем, что индексы элементов соответствующих групп Брауэра зависят только от поля определения, экспоненты и ветвления заданного элемента. Более точно, мы показываем, что на самом деле индекс элемента зависит не от ветвления в данной точке, а лишь от самой точки, в которой этот элемент ветвится. Этот результат позволяет нам получить оценки для индексов, лучшие, чем оценки, возникающие из результата ван ден Берга. Среди более ранних результатов по группам Брауэра полей функций проективных пространств, из которых легко извлекаются оценки для индексов их элементов (впрочем, не очень точные), следует упомянуть работу [9] о группе Брауэра комплексного проективного пространства. Отметим также работы [10], [11], в которых получено описание неразветвленных элементов экспоненты 2 групп Брауэра полей функций специальных кривых, и [12], [13], описывающие групшы Брауэра специальных поверхностей.

В статье мы последовательно рассматриваем случаи проективной прямой, проективного пространства и, наконец, случай гладкой проективной кривой.

\section{§2. Предварительные результаты}

Приведем вначале необходимые нам впоследствии обозначения и соглашения.

Пусть $k$ - произвольное поле характеристики нуль, содержащее примитивный корень степени $n$ из единицы, $X$ - гладкое проективное многообразие над $k$, $K=k(X)$ - его поле функций. Для кольца дискретного нормирования $R$ с полем вычетов $k(R)$ такого, что поле частных $R$ совпадает с $K$, сушествует гомоморфизм ветвления в $R$

$$
\partial_{R}: \operatorname{Br} K \rightarrow \operatorname{Hom}_{\text {cont }}\left(G_{R}, \mathbb{Q} / \mathbb{Z}\right)=H^{1}\left(G_{R}, \mathbb{Q} / \mathbb{Z}\right)
$$


где $G_{R}=\operatorname{Gal}(\bar{k} / k(R)), \bar{k}$ - алгебраическое замькание поля $k$ (см. [14]). Центральная простая алгебра (ц.п.а.) $A$ над $K$ называется разветвленной в $R$, если $\partial_{R}([A]) \neq 0$; в этом случае $R$ называется точкой ветвления. Известно, что число точек ветвления у ц.п.а. конечно. Подгруппа $\bigcap_{R} \operatorname{ker} \partial_{R}$, где $R$ пробегает множество колец с вышеуказанными свойствами, называется неразветвленной группой Брауәра поля $K$ и обозначается $\mathrm{Br}_{n r} K$.

Гомоморфизм $\partial_{R}$ устроен следующим образом (см. [14]). Пусть $R^{\prime}$ и $K^{\prime}$ - соответственно пополнение кольца $R$ и поля $K$. Тогда $R^{\prime}$ и $K^{\prime}$ могут быть отождествлены соответственно с кольцом $k(R)[[\pi]]$ и полем $k(R)((\pi))$. Пусть $K_{n r}^{\prime}$ - максимальное неразветвленное расширение поля $K^{\prime}$. Тогда $K_{n r}^{\prime}$ совпадает с полем $\bar{k}((\pi))$, $\operatorname{Gal}\left(K_{n r}^{\prime} / K^{\prime}\right)=G_{R}$ и $\overline{K^{\prime}}$ есть объединение всех полей вида $\bar{k}\left(\left(\pi^{1 / n}\right)\right)$. Групша Галуа $\operatorname{Gal}\left(\overline{K^{\prime}} / K_{n r}^{\prime}\right)$ тогда изоморфна групше $\lim \mathbb{Z} / n$. Когомологическая размерность этой группы равна 1 ; следовательно, $H^{2}\left(\operatorname{Gal}\left(\overline{K^{\prime}} / K_{n r}^{\prime}\right),{\overline{K^{\prime}}}^{*}\right)=0$. Так как $H^{1}\left(\mathrm{Gal}\left(\overline{K^{\prime}} / K_{n r}\right), \overline{K^{\prime}}{ }^{*}\right)=0$ по теореме Гильберта 90 , то последовательность ограничения и инфляции дает изоморфизм

$$
H^{2}\left(\operatorname{Gal}\left(K_{n r}^{\prime} / K^{\prime}\right), K_{n r}^{\prime}{ }^{*}\right) \cong H^{2}\left(\operatorname{Gal}\left(\overline{K^{\prime}} / K^{\prime}\right), \overline{K^{\prime}}{ }^{*}\right) .
$$

Нормирование $v: K^{\prime} \rightarrow \mathbb{Z}$ естественно продолжается до нормирования $v: K_{n r}^{\prime} \rightarrow \mathbb{Z}$, которое является гомоморфизмом $\operatorname{Gal}\left(K_{n r}^{\prime} / K^{\prime}\right)$-модулей $(\mathbb{Z}$ рассматривается как дискретный модуль). Тогда получаем гомоморфизм

$$
H^{2}\left(\operatorname{Gal}\left(K_{n r}^{\prime} / K^{\prime}\right), K_{n r}^{\prime}{ }^{*}\right) \rightarrow H^{2}\left(\operatorname{Gal}\left(K_{n r}^{\prime} / K^{\prime}\right), \mathbb{Z}\right)=H^{2}\left(G_{R}, \mathbb{Z}\right)
$$

Точная последовательность $G_{R}$-модулей $0 \rightarrow \mathbb{Z} \rightarrow \mathbb{Q} \rightarrow \mathbb{Q} / \mathbb{Z} \rightarrow 0$ дает изоморфизм $H^{2}\left(G_{R}, \mathbb{Z}\right) \cong H^{1}\left(G_{R}, \mathbb{Q} / \mathbb{Z}\right)=\operatorname{Hom}_{\text {cont }}\left(G_{R}, \mathbb{Q} / \mathbb{Z}\right)$.

Комбинируя изоморфизм (1) и гомоморфизм (2), получаем гомоморфизм

$$
\partial_{R^{\prime}}: \operatorname{Br} K^{\prime} \rightarrow \operatorname{Hom}_{\text {cont }}\left(G_{R}, \mathbb{Q} / \mathbb{Z}\right) .
$$

Тогда гомоморфизм $\partial_{R}$ определяется как композиция

$$
\operatorname{Br} K \stackrel{\text { res }}{\longrightarrow} \operatorname{Br} K^{\prime} \stackrel{\partial_{R^{\prime}}}{\longrightarrow} \operatorname{Hom}_{\text {cont }}\left(G_{R}, \mathbb{Q} / \mathbb{Z}\right) .
$$

Если $K$ - поле, содержащее примитивный корень $\rho$ степени $n$ из единицы, $a, b \in F^{*}$, то символ-алгеброй $(a, b)_{n}$ назьвается циклическая алгебра, порожденная над $K$ такими элементами $\alpha, \beta$, что $\alpha^{n}=a, \beta^{n}=b, \alpha \beta=\rho \beta \alpha$, элемент групшы Брауэра, содержащий алгебру $(a, b)_{n}$, будет обозначаться $\left[(a, b)_{n}\right]$.

Используя описание гомоморфизма $\partial_{R}$, нетрудно доказать следуюшее утверждение.

ЛЕмма 1. Пусть $\pi$, а и нормирования, связанного с кольцом $R$. Тогда

(i) $\partial_{R}\left(\left[(a, \pi)_{n}\right]\right)=0$ тогда и только тогда, когда $a \in\left(K^{*}\right)^{n}$;

(ii) $\partial_{R}\left(\left[(a, b)_{n}\right]\right)=0$.

Заметим, что в случае проективного пространства $\mathbb{P}_{k}^{m}$ существует взаимно однозначное соответствие между неприводимыми унитарными в смысле лексико-графического порядка многочленами и локальными кольцами $R$ конечных точек коразмерности 1. 


\section{§3. Случай проективной прямой и $P_{n, r}$-поля}

Рассмотрим сначала случай, когда $X$ - проективная прямая $\mathbb{P}_{k}^{1}$. В этом случае $\operatorname{Br} k=\operatorname{Br}_{n r} k\left(\mathbb{P}_{k}^{1}\right)$. Пусть $k\left(\mathbb{P}_{k}^{1}\right)=k(x)$. Если $\mathscr{A}$ - ц.п. $k\left(\mathbb{P}_{k}^{1}\right)$-алгебра и $f(x) \in K[x]$ - неприводимый унитарньй многочлен, определяющий нормирование поля $k\left(\mathbb{P}_{k}^{1}\right)$, то пусть $\mathscr{A}_{f(x)}=\mathscr{A} \otimes k(\theta)((f))$, где $\theta$ - корень многочлена $f(x)$ и $k(\theta)((f(x)))$ - поле формальных степенных рядов (пополнение поля $k\left(\mathbb{P}_{k}^{1}\right)$ по нормированию, определяемому многочленом $f(x))$.

Кроме того, ветвление алгебр удовлетворяет следующему закону взаимности Д. К. Фаддеева.

ПРЕДЛОЖЕНИЕ 2 [15], [16; III, Prop. 2.1], [17; § 1.2]. Существует следующая точная последовательность

$$
0 \longrightarrow \operatorname{Br} k \longrightarrow \operatorname{Br} k\left(\mathbb{P}_{k}^{1}\right) \stackrel{\oplus \partial_{R}}{\longrightarrow} \bigoplus_{R} H^{1}\left(G_{R}, \mathbb{Q} / \mathbb{Z}\right) \stackrel{\text { cor }}{\longrightarrow} H^{1}(\operatorname{Gal}(\bar{k} / k), \mathbb{Q} / \mathbb{Z}) \longrightarrow 0
$$

(здесь $R$ пробегает множсество таких колец, дискретного нормирования, что их поля частных совпадают с $k\left(\mathbb{P}_{k}^{1}\right)$, а сог суммирует локальнье гомоморфизмьг коограничения).

Заметим, что из закона взаимности Фаддеева следует, что если две ц.п.а. над $\left(\mathbb{P}_{k}^{1}\right)$ имеют одинаковое ветвление в кольцах, соответствующих конечным точкам проективной прямой, то они имеют одинаковое ветвление и в кольце, соответствующем бесконечной точке. Кроме того, если $\mathscr{A}$ не разветвлена, то $\mathscr{A}$ подобна некоторой константной ц.п.а., т.е. алгебре вида $\mathscr{B} \otimes_{k} k\left(\mathbb{P}_{k}^{1}\right)$, где $\mathscr{B}$ - ц.п.а. над $k$.

Нам также потребуется следующее

ОПРЕДЕЛЕНИЕ 3. Две ц.п. $k\left(\mathbb{P}_{k}^{1}\right)$-алгебры $\mathscr{A}$ и $\mathscr{B}$ называются фаддеевски әквивалентными, если найдется ц.п. $k$-алгебра $\mathscr{C}$ такая, что

$$
\mathscr{A} \sim B \otimes_{k\left(\mathbb{P}_{k}^{1}\right)}\left(\mathscr{C} \otimes_{k} k\left(\mathbb{P}_{k}^{1}\right)\right) .
$$

Ясно, что это - отношение эквивалентности. Алгебры $\mathscr{A}$ и $\mathscr{B}$ фаддеевски эквивалентны тогда и только тогда, когда они имеют одинаковое ветвление.

Наш основной результат в случае проективной прямой основан на следующей конструкции. Как уже отмечалось, все конечные точки ветвления алгебры $\mathscr{A}$ соответствуют неприводимым унитарным многочленам $f_{i}(x)$ над $k$. Пусть $F(x)$ есть произведение всех таких многочленов $f_{i}(x)$. Наш первый результат - следуюшая

ТЕОРЕМА 4. Пусть $k$ - поле, содержсащее примитивный корень $n$-й степени из единицы, $\mathscr{A}$ - и.п.а. над $k\left(\mathbb{P}_{k}^{1}\right)$ әкспоненты $n$. Тогда $\mathscr{A}$ фаддеевски эквивалентна и.п.а. индекса, не превосходящего $n^{[(\operatorname{deg} F(x)+1) / 2]}$, әде $[(\operatorname{deg} F(x)+1) / 2]$ - иелая часть числа $(\operatorname{deg} F(x)+1) / 2$.

ДоказАтельство. Пусть $m=[(\operatorname{deg} F(x)+1) / 2]$. Докажем утверждение теоремы индукцией по $m$. Если $m=0$, то $\operatorname{deg} F(x)=0$ и $\mathscr{A}$ не имеет ветвления в конечных точках. Тогда по закону взаимности $Ф$ аддеева алгебра $\mathscr{A}$ не разветвлена. Таким образом, $\mathscr{A}$ фаддеевски эквивалентна тривиальной алгебре.

Предположим, что утверждение теоремы верно при $m<s$. Рассмотрим случай $m=s$. Заметим, что при $\operatorname{deg} F(x)=1$ алгебра $\mathscr{A}$ фаддеевски эквивалентна 
алгебре вида $(u, x-a)_{n}$, где $F(x)=x-a$ и $u \in k^{*}$. Таким образом, будем считать, что $\operatorname{deg} F(x)>1$.

Сначала предположим, что $F(x)$ есть произведение линейных многочленов. Пусть $\left(x-a_{1}\right)\left(x-a_{2}\right)$ делит $F(x)$. Тогда

$$
\mathscr{A}_{x-a_{i}} \sim \mathscr{B}_{i} \otimes\left(u_{i}, x-a_{i}\right)_{n} \otimes_{k\left(\mathbb{P}_{k}^{1}\right)} k\left(\left(x-a_{i}\right)\right), \quad i=1,2
$$

где $u_{i}$ - ненулевой элемент поля $k$, а $\mathscr{B}_{i}-$ некоторая ц.п.а. над $k$. Тогда алгебра

$$
\mathscr{C}=\mathscr{A} \otimes_{k\left(\mathbb{P}_{k}^{1}\right)}\left(\left(x-a_{1}\right) u_{2}^{-1} /\left(a_{2}-a_{1}\right),\left(x-a_{2}\right) u_{1} /\left(a_{1}-a_{2}\right)\right)_{n}
$$

не разветвлена в многочленах $x-a_{1}$ и $x-a_{2}$. Действительно,

$$
\begin{aligned}
\mathscr{C}_{x-a_{1}} & =\mathscr{B}_{1} \otimes\left(u_{1}, x-a_{1}\right)_{n} \otimes\left(\left(x-a_{1}\right) u_{2}^{-1} /\left(a_{2}-a_{1}\right), u_{1}\right)_{n} \\
& \sim \mathscr{B}_{1} \otimes\left(u_{1}, x-a_{1}\right)_{n} \otimes\left(x-a_{1}, u_{1}\right)_{n} \otimes\left(u_{2}^{-1} /\left(a_{2}-a_{1}\right), u_{1}\right)_{n} \\
& \sim \mathscr{B}_{1} \otimes\left(u_{1}, x-a_{1}\right)_{n} \otimes\left(u_{1}^{-1}, x-a_{1}\right)_{n} \otimes\left(u_{2}^{-1} /\left(a_{2}-a_{1}\right), u_{1}\right)_{n} \\
& \sim \mathscr{B}_{1} \otimes\left(u_{2}^{-1} /\left(a_{2}-a_{1}\right), u_{1}\right)_{n} .
\end{aligned}
$$

Аналогично показывается, что

$$
\mathscr{C}_{x-a_{2}} \sim \mathscr{B}_{2} \otimes\left(u_{2}^{-1}, u_{1} /\left(a_{1}-a_{2}\right)\right)_{n}
$$

Таким образом, все конечные точки ветвления алгебры $\mathscr{C}$ соответствуют неприводимым унитарным делителям многочлена $F(x) /\left(\left(x-a_{1}\right)\left(x-a_{2}\right)\right)$. Так как

$$
\left[\left(\operatorname{deg}\left(F(x) /\left(\left(x-a_{1}\right)\left(x-a_{2}\right)\right)\right)+1\right) / 2\right]=[(\operatorname{deg} F(x)-2+1) / 2]=s-1,
$$

то по индуктивному предположению алгебра $\mathscr{C}$ фаддеевски эквивалентна алгебре индекса не большего, чем $n^{s-1}$, а тогда $\mathscr{A}$ фаддеевски эквивалентна алгебре индекса, не большего, чем $n^{s}$.

Теперь предположим, что $F(x)$ имеет неприводимый унитарньй делитель $f(x)$ степени, большей, чем 1.

Имеем

$$
\mathscr{A}_{f(x)}=\mathscr{A} \otimes_{k\left(\mathbb{P}_{k}^{1}\right)} k\left(\mathbb{P}_{k}^{1}\right)_{f(x)} \sim \mathscr{B} \otimes_{k\left(\mathbb{P}_{k}^{1}\right)_{f(x)}}(\alpha, f(x))_{n}
$$

где $\alpha$ - некоторый ненулевой элемент поля $k(\theta), \theta$ - корень многочлена $f(x)$ и $\mathscr{B}-$ ц.п.а. над $k(\theta)$. Найдется многочлен $g(x) \in k[x]$ такой, что $\alpha=g(\theta)$ и $\operatorname{deg} g(x)<$ $\operatorname{deg} f(x)$.

Тогда $\mathscr{C}=\mathscr{A} \otimes_{k\left(\mathbb{P}_{k}^{1}\right)}\left(g(x)^{-1}, f(x)\right)_{n}$ не разветвлена в $f(x)$. Если $\operatorname{deg} g(x)=0$, то конечные точки ветвления последней алгебры соответствуют неприводимьм унитарным делителям многочлена $F(x) / f(x)$. Так как $[(\operatorname{deg}(F(x) / f(x))+1) / 2]<s$, то последняя алгебра фаддеевски эквивалентна алгебре индекса, не превосходящего $n^{s-1}$, а тогда $\mathscr{A}$ фаддеевски эквивалентна алгебре индекса, не большего, чем $n^{s}$.

В случае $\operatorname{deg} g(x)>0$ через $g_{0}(x)$ обозначим неприводимый унитарный делитель многочлена $g(x)$ положительной степени, $g(x)=g_{0}(x)^{m} g_{1}(x)$, где $g_{0}(x)$ и 
$g_{1}(x)$ взаимно просты. Если алгебра $\mathscr{A}$ имеет ветвление в многочлене $g_{0}(x)$, то конечные точки ветвления алгебры $\mathscr{C}$ соответствуют некоторым неприводимым унитарным делителям многочлена $F(x) g_{1}(x) / f(x)$. Так как $\operatorname{deg} g_{1}(x)<\operatorname{deg} g(x)<$ $\operatorname{deg} f(x)$, то $\left[\left(\operatorname{deg}\left(F(x) g_{1}(x) / f(x)\right)+1\right) / 2\right]<s$. Следовательно, $\mathscr{A}$ фаддеевски эквивалентна алгебре индекса, не большего, чем $n^{s}$.

Если же алгебра $\mathscr{A}$ не имеет ветвления в многочлене $g_{0}(x)$, то пусть $h(x)$ - остаток от деления $f(x)$ на $g_{0}(x)$. Тогда

$$
\mathscr{A} \otimes\left(g(x)^{-1}, f(x)\right)_{n} \otimes\left(g(x)^{-1}, h(x)^{-1}\right)_{n} \sim \mathscr{A} \otimes_{k\left(\mathbb{P}_{k}^{1}\right)}\left(g(x)^{-1}, f(x) h(x)^{-1}\right)_{n}
$$

и конечные точки ветвления последней алгебры соответствуют некоторьм неприводимым унитарным делителям многочлена $F(x) g_{1}(x) h(x) / f(x)$. Так как

$$
\operatorname{deg} g_{1}(x)+\operatorname{deg} h(x)<\operatorname{deg} g_{1}(x)+\operatorname{deg} g_{0}(x) \leqslant \operatorname{deg} g(x) \leqslant \operatorname{deg} f(x)-1,
$$

то $\left[\left(\operatorname{deg}\left(F(x) g_{1}(x) h(x) / f(x)\right)+1\right) / 2\right]<s$. Следовательно, $\mathscr{A}$ фаддеевски эквивалентна алгебре индекса, не большего, чем $n^{s}$. Теорема доказана.

ЗАмечАниЕ. Заметим, что в случае $n=2$ имеем $[(\operatorname{deg} F(x)+1) / 2]=g+1$, где $g$ - род гиперэллиптической кривой, соответствующей уравнению $y^{2}=F(x)$.

Таким образом, в случае $n=2$ предыдушая теорема может быть переформулирована следующим образом.

Tеорема 5. Пусть $\mathscr{A}$ - и.п.а. над $k\left(\mathbb{P}_{k}^{1}\right)$ әкспоненты 2. Тогда $\mathscr{A}$ фаддеевски әквивалентна и.п.а. индекса, не превосходящего $n^{g+1}$, где $g$ - род гиперәллиптической кривой, соответствующей уравнению $y^{2}=F(x)$.

ЗАмечАнИЕ. Доказательство теоремы 4 может быть использовано для получения следующего результата, полученного С. Блохом (см. [18]).

Теорема 6. Пусть $k$ - поле, содержащее группу $\mu_{n}$ корней $n$-й степени из $1, L=k\left(x_{1}, \ldots, x_{s}\right)$ - поле функций пространства $\mathbb{P}_{k}^{s}$. Тогда естественные гомоморфизмы, индуцированные гомоморфизмом норменного вычета,

$$
\begin{aligned}
& R_{n, k}: K_{2}(k) / n K_{2}(k) \rightarrow{ }_{n} \operatorname{Br} k, \\
& R_{n, L}: K_{2}(L) / n K_{2}(L) \rightarrow{ }_{n} \operatorname{Br} L
\end{aligned}
$$

имеют изоморфные коядра.

ДокАЗАТЕЛЬСтво. Нетрудно видеть, что достаточно доказать утверждение теоремы в случае $L=k(x)$. Построим гомоморфизм

$$
\varphi:{ }_{n} \operatorname{Br} k / \operatorname{im}\left(R_{n, k}\right) \rightarrow{ }_{n} \operatorname{Br} k(x) / \operatorname{im}\left(R_{n, k(x)}\right),
$$

положив $\varphi\left(a+\operatorname{im}\left(R_{n, k}\right)\right)=\operatorname{res}_{k(x) / k}(a)+\operatorname{im}\left(R_{n, k(x)}\right)$. Проверим корректность. Пусть $a \in \operatorname{im}\left(R_{n, k}\right)$, тогда $a$ представляется произведением символ-алгебр, следовательно, $\operatorname{res}_{k(x) / k}(a)$ представляется произведением символ-алгебр и $\operatorname{res}_{F / k}(a) \in$ $\operatorname{im}\left(R_{n, F}\right)$. Корректность доказана.

Установим инъективность гомоморфизма $\varphi$. Пусть $\operatorname{res}_{k(x) / k}(a) \in \operatorname{im}\left(R_{n, k(x)}\right)$. Следовательно, $\operatorname{res}_{k(x) / k}(a)$ представляется произведением символ-алгебр. Тогда 
$\operatorname{res}_{k(x)_{\infty} / k}(a)$ представляется произведением символ-алгебр, где $k(x)_{\infty}$ - пополнение поля $k(x)$ по нормированию в бесконечной точке. Откуда $a \in \operatorname{im}\left(R_{n, k}\right)$. Таким образом, инъективность доказана. Докажем сюръективность. Любой элемент $b$ из ${ }_{n} \operatorname{Br} F$ представляется алгеброй вида $\left(\mathscr{A} \otimes_{k} k(x)\right) \otimes\left(f_{i}, g_{i}\right)$, где $\mathscr{A}$ - некоторая алгебра над $k$, а $f_{i}, g_{i} \in k(x)$. Тогда $b+\operatorname{im}\left(R_{n, k(x)}\right)$ есть образ $\mathscr{A}+\operatorname{im}\left(R_{n, k}\right)$. Следовательно, коядра изоморфны. Теорема доказана.

В качестве простого следствия из предыдущей теоремы получаем, что ц.п.а. экспоненты $n$ над $k\left(x_{1}, \ldots, x_{s}\right)$ подобна тензорному произведению циклических алгебр тогда и только тогда, когда это справедливо для любой ц.п. $k$-алгебры экспоненты, делящей $n$. Это дает в случае специальных полей $k$ элементарное доказательство теоремы Меркурьева-Суслина для полей $k\left(x_{1}, \ldots, x_{s}\right)$.

Теорема 4 дает верхние оценки для индексов ц.п. алгебр экспоненты $n$ над $k\left(\mathbb{P}_{k}^{1}\right)$ по модулю индексов ц.п. $k$-алгебр экспонент, делящих $n$. В случае специальных классов полей констант $k$ теорема 4 дает оценки для индексов ц.п. алгебр экспоненты $n$ над $k\left(\mathbb{P}_{k}^{1}\right)$. Важным в этом отношении является класс $P_{n, r}$-полей.

ОПРЕДЕЛЕНИЕ 7 . Пусть $n, r$-целые неотрицательные числа, $n>0$. Поле $k$ называется $P_{n, r}$-полем, если для любой $k$-алгебры $\mathscr{A}$ экспоненты, делящей $n, i(\mathscr{A})$ делит $n^{r}$.

Заметим, что если $k-P_{n, r}$-поле, то $k-P_{n, s}$-поле для любого $s>r$.

Для удобства дальнейшего изложения приведем несколько элементарных свойств $P_{n, r}$-полей.

Лемма 8. Пусть $n=p_{1}^{\alpha_{1}} \cdots p_{s}^{\alpha_{s}}$, где $p_{i}-$ различные простые числа и $\alpha_{i} \geqslant 1$. Ecли $k-P_{n, r}$-noлe, mo $k-P_{p_{i}^{\alpha_{i}, r}}$-noлe.

ДокАЗАТЕльство. Пусть $\mathscr{A}$ - любая алгебра экспоненты, делящей $p_{i}^{\alpha_{i}}$. Тогда $i(\mathscr{A})$ делит $n^{r}$. Следовательно, $i(\mathscr{A})$ делит $p_{i}^{\alpha_{i} r}$.

Лемма 9. Пусть $n=p_{1}^{\alpha_{1}} \cdots p_{l}^{\alpha_{l}}$, әде $p_{i}$ - различнье простье числа $и \alpha_{i} \geqslant 1$. Ecли $k-P_{p_{i}^{\alpha_{i}, s_{i}}}{ }^{-n o л e, ~ m o ~} k-P_{n, r}-$ noлe, где $r=\operatorname{HOK}\left(s_{1}, \ldots, s_{l}\right)$.

ДоказАтельство. Пусть $e(\mathscr{A})$ делит $n$ и $\mathscr{A}=\mathscr{A}_{1} \otimes \cdots \otimes \mathscr{A}_{s}-$ разложение алгебры $\mathscr{A}$ в тензорное произведение алгебр экспонент, делящих $p_{i}^{\alpha_{i}}$. Тогда $i(\mathscr{A})=$ $\prod_{i} i\left(\mathscr{A}_{i}\right)$ и $i\left(\mathscr{A}_{i}\right)$ делит $p_{i}^{\alpha_{i} s_{i}}$. Следовательно, $i(\mathscr{A})$ делит $\prod_{i} p_{i}^{\alpha_{i} s_{i}}$. Тогда $i(\mathscr{A})$ делит $\prod_{i} p_{i}^{\alpha_{i} r}$.

Предыдущие леммы позволяют при изучении свойства $P_{n, r}$ ограничиться примарным $n$. Ниже $n=p^{m}$, где $p$ - простое.

ЛЕмма 10. Пусть $k / F-$ конечное расширение полей и $k-P_{n, r}$-поле. Тогда $F-P_{n, s}$-поле, где $s=\left[\log _{n}[k: F]_{p}\right]+1+r,[k: F]_{p}-p$-часть числа $[k: F]$.

ДокАЗАтЕльство. Пусть $e(\mathscr{A})$ делит $n$. Тогда $e\left(\mathscr{A} \otimes_{F} k\right)$ делит $n$. Тогда $i\left(\mathscr{A} \otimes_{F} k\right)$ делит $n^{r}$ и $i(\mathscr{A})$ делит $[k: F]_{p} n^{r}$. Следовательно, $F-P_{n, s}$-поле, где $s=\left[\log _{n}[k: F]_{p}\right]+1+r$. 
ЗАмечАниЕ. Последняя лемма дает полезный способ построения новых $P_{n, r^{-}}$ полей из имеющихся.

В заключение приведем список (далеко не полный) некоторых полей указанного типа.

(i) Алгебраически замкнутые поля и конечные поля являются $P_{n, 0}$-полями.

(ii) Вешественно замкнутые поля $-P_{2,1}$-поля и $P_{n, 0}$-поля, если $n$ нечетно.

(iii) Поле функций кривой над алгебраически замкнутым полем $-P_{n, 0}$-поле.

(iv) Поле функций кривой над вешественно замкнутым полем - $P_{2,1}$-поле и $P_{n, 0^{-}}$ поле, если $n$ нечетно.

(v) Поле функций локальной кривой $-P_{n, 2}$-поле в случае, когда $n$ взаимно просто с характеристикой поля вычетов (см. [6], [7]).

(vi) Пусть $R$-гензелево дискретно нормированное кольцо с полем частных $K$ и полем вычетов $k$. Предположим, что $n$ взаимно просто с характеристикой поля $k$ и группа Брауэра любой проективной кривой над всяким конечным расширением поля $k$ тривиальна. Тогда для любой кривой $C$ над $K$ поле $K(C)$ является $P_{n, 2}$-полем (см. [6], [7]).

(vii) Пусть $k$ - поле с гензелевым нормированием ранга $m$ и полем вычетов нулевой характеристики, являющимся $P_{n, r}$-полем. Тогда $k-P_{n, m+r}$-поле.

(viii) Пусть $R$ - превосходное гензелево локальное кольцо размерности два, $K-$ его поле частных и $k$ - поле вычетов. Предположим, что $k$ сепарабельно замкнуто и $n$ взаимно просто с характеристикой поля $k$. Тогда $K-P_{n, 1}$-поле (см. [4]).

(ix) $C_{2}$-поле является $P_{n, 1}$-полем, при $n \in\{2,3\}$.

$(\mathrm{x})$ Пусть $k$ содержит примитивный корень из 1 степени $n$, взаимно простой с характеристикой $k$, и группа $k^{*} /\left(k^{*}\right)^{n}$ конечна. Тогда $k$ является $P_{n, r}$-полем при некотором $r$.

Для $P_{n, r}$-полей теорема 4 может быть переформулирована следующим образом.

ТЕОРема 11. Пусть $k-P_{n, r}$-поле, содержащее примитивный корень $n$-й степени из $1, \mathscr{A}$ - и.п.а. над $k\left(\mathbb{P}_{k}^{1}\right)$ әкспоненты $n$ и $F(x)$ - то жее, что $и$ выцие. Тогда $i(\mathscr{A}) \leqslant n^{r+[(\operatorname{deg} F+1) / 2]}$.

Вообще говоря, предыдущая оценка не является оптимальной, что следует, например, из результата Д. Солтмэна (см. [6], [7]), где $k$ - конечное расширение поля $\mathbb{Q}_{p}$.

Однако ситуация не так проста, если рассматривать весь класс $P_{n, r}$-полей. Более того, нам кажется, что следующий вопрос имеет смысл.

ВоПрос. Пусть $n, r$ - натуральные числа. Существует ли такое $P_{n, r^{-}}$ поле, содержащее примитивный корень $n$-й степени из 1 , что оценка, полученная в теореме 11, достигается.

\section{§4. Случай проективного пространства}

Теорема 4 может быть обобщена на случай проективного пространства.

Теорема 12. Пусть $k-P_{n, r}$-поле, содержащее примитивный корень $n$-й степени из $1, L=k\left(\mathbb{P}_{k}^{s}\right)=k\left(x_{1}, \ldots, x_{s}\right), \mathscr{A}-$ и.n.а. над L әкспоненты $n$ с ветвлением в многочленах $F_{1}\left(x_{1}, \ldots, x_{s}\right), \ldots, F_{t}\left(x_{1}, \ldots, x_{s}\right)$. Пусть $G\left(x_{1}, \ldots, x_{s}\right)=$ 
$F_{1}\left(x_{1}, \ldots, x_{s}\right) \cdots F_{t}\left(x_{1}, \ldots, x_{s}\right)$ u $d_{j}$ - степень многочлена $G\left(x_{1}, \ldots, x_{s}\right)$ по переменной $x_{j}$. Тогда

$$
i(\mathscr{A}) \leqslant n^{r+M_{1}+\cdots+M_{s}+\min _{i}\left\{\sum_{1 \leqslant j \leqslant s, j \neq i} M_{j}\right\}}
$$

əде $M_{i}=\left[\left(d_{i}+1\right) / 2\right]$.

Доказательство теоремы предварим двумя леммами.

Лемма 13. Пусть $\mathscr{A}-$ u.n.а. над $L=k\left(\mathbb{P}_{k}^{s}\right)=k\left(x_{1}, \ldots, x_{s}\right)$ с конечнымм ветвлением в неприводимых многочленах $F_{1}\left(x_{1}, \ldots, x_{s}\right), \ldots, F_{t}\left(x_{1}, \ldots, x_{s}\right)$. Тогда конечное ветвление алгебры $\mathcal{A}$, рассматриваемой как алгебры над $k\left(x_{1}, \ldots, x_{s-1}\right)\left(\mathbb{P}_{k}^{1}\right)=k\left(x_{1}, \ldots, x_{s-1}\right)\left(x_{s}\right)$ (т.е. над полем рациональных функций $c$ полем констант $\left.k\left(x_{1}, \ldots, x_{s-1}\right)\right)$, сосредоточено в некоторых из унитарных многочленов, являющихся делителями многочлена $F_{1}\left(x_{1}, \ldots, x_{s}\right) \cdots F_{t}\left(x_{1}, \ldots, x_{s}\right)$, рассматриваемого как многочлен из $k\left(x_{1}, \ldots, x_{s-1}\right)\left[x_{s}\right]$.

ДоказАтельство. Рассмотрим $\mathscr{A}$ как алгебру над $k\left(x_{1}, \ldots, x_{s-1}\right)\left(x_{s}\right)$. Пусть $H\left(x_{s}\right) \in k\left(x_{1}, \ldots, x_{s-1}\right)\left[x_{s}\right]$ - унитарньй неприводимый многочлен, в котором $\mathscr{A}$ имеет ветвление. Тогда

$$
H\left(x_{s}\right)=x_{s}^{n}+\left(u_{n-1} / v_{n-1}\right) x_{s}^{n-1}+\cdots+u_{0} / v_{0}
$$

где $u_{i}, v_{i} \in k\left[x_{1}, \ldots, x_{s-1}\right]$ и $u_{i} / v_{i}-$ несократимые дроби.

Алгебра $\mathscr{A}$ подобна произведению некоторых циклических алгебр, т.е.

$$
\mathscr{A} \sim\left(H\left(x_{s}\right), G\right)_{l} \otimes \mathscr{B}
$$

где $G$ - некоторый элемент кольца $k\left(x_{1}, \ldots, x_{s-1}\right)\left[x_{s}\right]$, а $\mathscr{B}$ есть произведение символ-алгебр, не содержащих элемент $H\left(x_{s}\right)$. В $H\left(x_{s}\right)$ алгебра $\mathscr{A}$ имеет ветвление тогда и только тогда, когда

$$
G(\theta) \notin\left(k\left(x_{1}, \ldots, x_{s-1}\right)(\theta)^{*}\right)^{l}
$$

где $\theta$ - корень многочлена $H\left(x_{s}\right)$ в алгебраическом расширении поля $k\left(x_{1}, \ldots\right.$, $x_{s-1}$ ) (см. лемма 1). Покажем, что

1 ) многочлен $T=H\left(x_{s}\right) \operatorname{HOK}\left(v_{0}, \ldots, v_{n-1}\right)$ неприводим над $k$;

2) многочлен $T$ содержит ветвление алгебры $\mathscr{A}$ как алгебры над $k\left(x_{1}, \ldots, x_{s}\right)$ с полем констант $k$.

1) Предположим, что $T$ приводим, тогда $T=T_{1} T_{2}$, где $T_{i} \in k\left[x_{1}, \ldots, x_{s}\right]$. Если $T_{1}$ и $T_{2}$ содержат переменную $x_{s}$, то многочлен $H\left(x_{s}\right)$ приводим как многочлен от $x_{s}$. Таким образом, можно считать, что $T_{1}$ не содержит $x_{s}$. Но тогда множество коэффициентов многочлена $T \in k\left[x_{1}, \ldots, x_{s-1}\right]\left[x_{s}\right]$ обладает неприводимьп общим делителем из $k\left[x_{1}, \ldots, x_{s-1}\right]$. Заметим, что коэффициент многочлена $T$ при $x_{s}^{n}$ есть $\operatorname{HOK}\left(v_{0}, \ldots, v_{n-1}\right)$, а при $x_{s}^{i}$ равен

$$
\operatorname{HOK}\left(v_{0}, \ldots, v_{n-1}\right) u_{i} / v_{i}, \quad i=0, \ldots, n-1
$$


Пусть $S \in k\left[x_{1}, \ldots, x_{s-1}\right]$ - неприводимый делитель всех коэффициентов многочлена $T$. Тогда $S$ делитель $\operatorname{HOK}\left(v_{0}, \ldots, v_{n-1}\right)$. Следовательно, $S$ делит по крайней мере один $v_{i}$. Выберем многочлен $v_{j}$, делящийся на $S$ в максимально большой степени. Тогда $S$ делит $\operatorname{HOK}\left(v_{0}, \ldots, v_{n-1}\right) u_{j} / v_{j}$. Но по выбору $v_{j} S$ не делит $\operatorname{HOK}\left(v_{0}, \ldots, v_{n-1}\right) / v_{j}$. Тогда $S$ - делитель $u_{j}$, а следовательно, $u_{j}$ и $v_{j}$ имеют общий делитель. Противоречие с несократимостью $u_{j} / v_{j}$. Значит, $T$ неприводим.

2) Покажем, что $\mathscr{A}$ ветвится в $T$. Имеем $\mathscr{A} \sim\left(H\left(x_{m}\right), G\right)_{l} \otimes \mathscr{B}$. Тогда

$$
\begin{aligned}
\mathscr{A} & \sim\left(H\left(x_{s}\right) \operatorname{HOK}\left(v_{0}, \ldots, v_{n-1}\right) \operatorname{HOK}\left(v_{0}, \ldots, v_{n-1}\right)^{l-1}, G\right)_{l} \otimes \mathscr{B} \\
& =\left(\operatorname{HOK}\left(v_{0}, \ldots, v_{n-1}\right)^{l-1} T, G\right)_{l} \otimes \mathscr{B} .
\end{aligned}
$$

Заметим, что так как алгебра $\mathscr{B}$ - произведение символ-алгебр, не содержащих $H\left(x_{s}\right)$, то $\mathscr{B}$ не ветвится в $T$. Кроме того, $\left(\mathrm{HOK}\left(v_{0}, \ldots, v_{n-1}\right)\right)^{l-1}$ на $T$ не делится, так как не содержит переменной $x_{s}$. Итак, ветвление в $T$ определяется элементом $G$. Причем алгебра $\mathscr{A}$ ветвится в $T$ тогда и только тогда, когда $G \notin\left(k\left(C_{T}\right)^{*}\right)^{l}$, где $C_{T}$ - гиперповерхность, задаваемая уравнением $T=0$. Пусть $G \in\left(k\left(C_{T}\right)^{*}\right)^{l}$. Следовательно, найдутся такие многочлены $G_{1}, G_{2} \in$ $k\left[x_{1}, \ldots, x_{s}\right]$, что $G=G_{1}^{l}+G_{2} T$. Подставляя в последнее равенство вместо $x_{s}$ элемент $\theta$, получаем

$$
G(\theta)=G_{1}(\theta)^{l}+G_{2} T(\theta)=G_{1}(\theta)^{l} .
$$

Но это противоречит (3). Таким образом, алгебра $\mathscr{A}$ ветвится в $T$. Лемма доказана.

ЛЕмма 14. Пусть $k$ - поле нулевой характеристики, $H_{1}, \ldots, H_{m}-$ неприводимые попарно взаимно простые многочлень из $k\left[x_{1}, \ldots, x_{s}\right]$. Тогда найдется бесконечно много таких әлементов а поля $k$, что многочлень $\bar{H}_{i}=H_{i}\left(x_{1}, \ldots, x_{s-1}, a\right) \in k\left[x_{1}, \ldots, x_{s-1}\right]$ попарно взаимно просты.

ДоКАЗАТЕЛЬСтво. Пусть $Y_{i j} \subset \mathrm{A} \frac{s}{k}$ - множества, заданные системами уравнений $H_{i}=0$ и $H_{j}=0, i \neq j$. Тогда $\operatorname{dim} Y_{i j} \leqslant s-2$ и $\operatorname{dim} \bigcup_{i, j} Y_{i j} \leqslant s-2$. Рассмотрим $\bigcup_{i, j} Y_{i j} \cap X_{a}$, где $X_{a}$-гиперплоскость, заданная уравнением $x_{s}=a$. Предположим, что найдутся многочлены $H_{i}$ и $H_{j}$ такие, что $\bar{H}_{i}$ и $\bar{H}_{j}$ имеют общий делитель $h \in k\left[x_{1}, \ldots, x_{s-1}\right]$. Пусть множество $Z \subset \mathrm{A} \frac{s}{k}$ определяется уравнением $h=0$, где $h$ рассматривается как многочлен из $k\left[x_{1}, \ldots, x_{s}\right]$. Тогда

$$
Z \cap X_{a} \subset Y_{i j} \cap X_{a} \subset \bigcup_{i, j} Y_{i j} \cap X_{a} .
$$

Действительно, если $\bar{H}_{i}=h_{i} h$ и $\bar{H}_{j}=h_{j} h$, то $Y_{i j} \cap X_{a}$ задается уравнениями

$$
x_{s}=a, \quad h_{i} h=0, \quad h_{j} h=0 .
$$

Так как $Z \cap X_{a}$ задано уравнениями $x_{s}=a$ и $h=0$, то $Z \cap X_{a} \subset Y_{i j} \cap X_{a}$. Тогда

$$
s-2 \leqslant \operatorname{dim} Z \cap X_{a}=\operatorname{dim} Y_{i j} \cap X_{a}=\operatorname{dim} \bigcup_{i, j} Y_{i j} \cap X_{a}
$$

поскольку $\operatorname{dim} Y_{i j} \cap X_{a} \leqslant s-2$. Следовательно, $\operatorname{dim} \bigcup_{i, j} Y_{i j} \cap X_{a}=\operatorname{dim} \bigcup_{i, j} Y_{i j}=$ $s-2$. 
Так как $\operatorname{dim} \bigcup_{i, j} Y_{i j} \cap X_{a}=s-2$, то $X_{a}$ содержит неприводимую компоненту многообразия $\bigcup_{i, j} Y_{i j}$ размерности $s-2$. Заметим, что число таких компонент конечно.

Предположим, что для бесконечного множества $a \in k$ какие-то из многочленов $\bar{H}_{i}$ и $\bar{H}_{j}$ имеют общий делитель в $k\left[x_{1}, \ldots, x_{s-1}\right]$.

Тогда для таких $а$ гиперплоскость $X_{a}$ содержит неприводимую компоненту многообразия $\bigcup_{i, j} Y_{i j}$ размерности $s-2$. Так как число компонент конечно, то найдутся такие различные $a_{1}$ и $a_{2}$ из $k$, что $X_{a_{1}}$ и $X_{a_{2}}$ содержат одну и ту же компоненту размерности $s-2$. Но $X_{a_{1}} \cap X_{a_{2}}=\varnothing$. Полученное противоречие доказывает лемму.

ДОКАЗАТЕЛЬСТво ТЕОРЕМЫ 12. Применим индукцию по $s$. В случае $s=1$ утверждение следует из теоремы 11. Предположим, что оно справедливо при $s \leqslant$ $m-1$ и рассмотрим случай $s=m$.

Алгебра $\mathscr{A}$ является алгеброй над полем функций проективной прямой $\mathbb{P}_{k\left(x_{1}, \ldots, x_{m-1}\right)}^{1}$. В силу леммы 13 все конечные точки ветвления алгебры $\mathscr{A}$ соответствуют некоторым делителям многочлена $G\left(x_{1}, \ldots, x_{m}\right)$. Тогда по теореме 11 алгебра $\mathscr{A}$ фаддеевски эквивалентна над $k\left(x_{1}, \ldots, x_{m-1}\right)$ алгебре $\mathscr{B}$ индекса, не большего, чем $n^{\left[\left(d_{m}+1\right) / 2\right]}=n^{M_{m}}$.

Пусть $\mathscr{A} \sim \bigotimes_{i}\left(U_{i}, V_{i}\right)_{n}$, где многочлены $U_{i}, V_{i} \in k\left[x_{1}, \ldots, x_{m}\right]$ неприводимы и НОД $\left(U_{i}, V_{i}\right)=1$. Пусть $H_{i}, \ldots, H_{q}$ - все неприводимые попарно взаимно простые многочлены, присутствуюшие в символ-алгебрах из разложения $\mathscr{A}$. По лемме 14 найдется такое $a \in k$, что многочлены $\bar{H}_{i}$ взаимно простыми. Тогда $\mathscr{A}_{x_{m}-a} \sim$ $\bigotimes_{i}\left(\bar{U}_{i}, \bar{V}_{i}\right)_{n}$ и НОД $\left(\bar{U}_{i}, \bar{V}_{i}\right)=1$. Покажем, что отсутствие ветвления $\mathscr{A}$ в $H \in$ $\left\{H_{1}, \ldots, H_{q}\right\}$ влечет отсутствие ветвления $\mathscr{A}_{x_{m}-a}$ в $\bar{H}$. Перепишем $\mathscr{A}$ в виде

$$
\mathscr{A} \sim(H, U)_{n} \otimes\left(\bigotimes_{i}\left(S_{i}, T_{i}\right)_{n}\right),
$$

где НОД $(H, U)=$ НОД $\left(H, S_{i}\right)=$ НОД $\left(H, T_{i}\right)=1$. Тогда

$$
\mathscr{A}_{x_{m}-a} \sim(\bar{H}, \bar{U})_{n} \otimes\left(\bigotimes_{i}\left(\bar{S}_{i}, \bar{T}_{i}\right)_{n}\right)
$$

и согласно выбору а НОД $(\bar{H}, \bar{U})=$ НОД $\left(\bar{H}, \bar{S}_{i}\right)=$ НОД $\left(\bar{H}, \bar{T}_{i}\right)=1$. Так как $\mathscr{A}$ не ветвится в $H$, то $U \in\left(k\left(X_{H}\right)^{*}\right)^{n}$, где множество $X_{H} \subset \mathrm{A}_{\frac{m}{k}}$ определено уравнением $H=0$. Тогда найдутся такие $G_{1}, G_{2} \in k\left[x_{1}, \ldots, x_{m}\right]$, что $U=G_{1}^{n}+G_{2} H$, что влечет $\bar{U}=\bar{G}_{1}^{n}+\bar{G}_{2} \bar{H}$. Рассмотрим ветвление алгебры $\mathscr{A}_{x_{m}-a}$ в каком-нибудь неприводимом делителе $h_{0}$ многочлена $\bar{H}$. Ветвление в $h_{0}$ определяется элементом $\bar{U} \in k\left(Y_{h_{0}}\right)$, где $Y_{h_{0}} \subset A_{\bar{k}}^{m-1}$ задано уравнением $h_{0}=0$. Но так как $\bar{U}=\bar{G}_{1}^{n}+\bar{G}_{2} \bar{H}$ и $h_{0}$ делит $\bar{H}$, то $\bar{U} \in k\left(Y_{h_{0}}^{*}\right)^{n}$, т.е. ветвления в $h_{0}$ нет. Таким образом, если ветвление у $\mathscr{A}_{\text {в } H}$ отсутствует, то у $\mathscr{A}_{x_{m}-a}$ его нет в делителях $\bar{H}$. Следовательно, все конечное ветвление алгебры $\mathscr{A}_{x_{m}-a}$ сосредоточено в некоторых делителях многочленов $\bar{F}_{i}\left(x_{1}, \ldots, x_{m}\right)$.

Имеем

$$
\begin{aligned}
\mathscr{B}_{x_{m}-a} & =\mathscr{B} \otimes k\left(x_{1}, \ldots, x_{m-1}\right)\left(x_{m}\right)_{x_{m}-a} \\
& \sim \mathscr{C} \otimes_{k\left(x_{1}, \ldots, x_{m-1}\right)} k\left(x_{1}, \ldots, x_{m-1}\right)\left(x_{m}\right)_{x_{m}-a}
\end{aligned}
$$


где $\mathscr{C}$ - некоторая ц.п.а. над $k\left(x_{1}, \ldots, x_{m-1}\right)$. Тогда

$$
i\left(\mathscr{C} \otimes_{k\left(x_{1}, \ldots, x_{m-1}\right)} k\left(x_{1}, \ldots, x_{m-1}\right)\left(x_{m}\right)_{x_{m}-a}\right) \leqslant i(B) \leqslant n^{M_{m}}
$$

и

$$
\left(\mathscr{B} \otimes\left(\mathscr{C}^{-1} \otimes k\left(x_{1}, \ldots, x_{m}\right)\right)\right)_{x_{m}-a} \sim 1 .
$$

Рассмотрим алгебру $\mathscr{A}_{x_{m}-a}$. Имеем

$$
\mathscr{A}_{x_{m}-a} \sim \mathscr{D} \otimes k\left(x_{1}, \ldots, x_{m-1}\right)\left(x_{m}\right)_{x_{m}-a},
$$

где $\mathscr{D}$ - ц.п.а. над $k\left(x_{1}, \ldots, x_{m-1}\right)$. Из леммы 14 вытекает, что алгебра $\mathscr{D}$ может ветвиться только в некоторых неприводимых делителях многочлена $\bar{G}$. Заметим, что степень многочлена $\bar{G}$ по переменным $x_{j}$ не превосходит $d_{j}$. Следовательно, по индуктивному предположению

$$
i(\mathscr{D}) \leqslant n^{r+M_{1}+\cdots+M_{m-1}+\min _{1 \leqslant i \leqslant m-1}\left\{\sum_{1=j \neq i}^{m-1} M_{j}\right\}} .
$$

Далее, $\mathscr{A} \otimes\left(\mathscr{D}^{-1} \otimes k\left(x_{1}, \ldots, x_{m}\right)\right)_{x_{m}-a} \sim 1$. Следовательно, алгебры $\mathscr{A} \otimes$ $\left(\mathscr{D}^{-1} \otimes k\left(x_{1}, \ldots, x_{m}\right)\right)$ и $\mathscr{B} \otimes\left(\mathscr{C}^{-1} \otimes k\left(x_{1}, \ldots, x_{m}\right)\right)$ имеют тривиальное пополнение в $k\left(x_{1}, \ldots, x_{m-1}\right)$-рациональной точке. Таким образом,

$$
\mathscr{A} \sim \mathscr{B} \otimes\left(\mathscr{D} \otimes \mathscr{C}^{-1} \otimes L\right) .
$$

Тогда

$$
i(\mathscr{A}) \leqslant n^{r+M_{1}+\cdots+M_{m}+\min _{1 \leqslant i \leqslant m-1}\left\{\sum_{1=j \neq i}^{m-1} M_{j}\right\}} .
$$

Заметим, что $\mathscr{A}$ можно рассматривать как ц.п.а. над полем функций проективной прямой $\mathbb{P}_{k\left(x_{1}, \ldots, x_{l-1}, x_{l+1}, \ldots, x_{m}\right)}^{1}$. В этом случае оценка для индекса будет такова

$$
i(\mathscr{A}) \leqslant n^{r+M_{1}+\cdots+M_{m}+\min _{1 \leqslant i \leqslant m, i \neq l}\left\{\sum_{1=j \neq i}^{m-1} M_{j}\right\}} .
$$

Откуда получаем следующую оценку

$$
\begin{aligned}
i(\mathscr{A}) & \leqslant n^{r+M_{1}+\cdots+M_{m}+\min _{l}\left\{\min _{1 \leqslant i \leqslant m, i \neq l}\left\{\sum_{1=j \neq i}^{m-1} M_{j}\right\}\right\}} \\
& =n^{r+M_{1}+\cdots+M_{m}+\min _{1 \leqslant i \leqslant m}\left\{\sum_{1=j \neq i}^{m-1} M_{j}\right\}} .
\end{aligned}
$$

Теорема доказана.

\section{§5. Случай проективной кривой}

В этом параграфе мы рассматриваем случай произвольной гладкой проективной кривой $C$ над $k$ с полем функций $k(C)$. Полученные результаты основаны на следующей теореме ван ден Берга.

Теорема 15 [8]. Пусть $C$ - гладкая проективная кривая над $k$ с $k$-рачиональной точкой $P$ и $\mathscr{D}$ - неразветвленная алгебра над $k(C)$ такая, что $\mathscr{D} \otimes k(C)_{P}-$ полная матричная алгебра над $k(C)_{P}$, где $k(C)_{P}-$ пополнение поля $k(C)$ по нормированию, соответствующему точке $P$. Тогда $i(\mathscr{D}) \mid e(\mathscr{D})^{2 g(C)}$, где $g(C)-$ род кривой $C$.

Первый основной результат содержится в следуюшем утверждении. 
ТЕОРЕМА 16. Пусть $k$ и все конечные расширения поля $k$ являются $P_{n, r}-$ полями, $k$ содержст примитивный корень степени $n$ из $1, C$ - гладкая проективная кривая над $k, \mathscr{A}$ - чентральная простая алгебра над $k(C)$ әкспоненmвь $n=p_{1}^{n_{1}} \cdots p_{q}^{n_{q}}$ с ветвлением в замкнутых точках $P_{1}, \ldots, P_{s}$ кривой $C$, $L=k\left(P_{1}, \ldots, P_{s}\right)-$ поле определения этих точек $u t=p_{1}^{m_{1}} \cdots p_{q}^{m_{q}} u-$ степень расиирения $L$ над $k$, где НОД $(n, u)=1$. Тогда

$$
i(A) \leqslant n^{r+N+2 g(C)} p_{1}^{m_{1}} \cdots p_{q}^{m_{q}},
$$

где $N$ - число точек поля $\bar{k}(C)$ над точками $P_{i}$.

ДоказАтельство. Алгебра $\mathscr{A} \otimes_{k(C)} L(C)$ имеет ветвление только в $N L$-рациональных точках. Ветвление в этих точках определяется элементами $a_{1}, \ldots, a_{N}$ поля $L^{*}$. Тогда алгебра $\mathscr{B}=\mathscr{A} \otimes L\left(\sqrt[n]{a_{1}}, \ldots, \sqrt[n]{a_{N}}\right)$ не разветвлена. Следовательно, по теореме ван ден Берга $i\left(\mathscr{B} \otimes \mathscr{B}_{P}^{n-1}\right) \leqslant n^{2 g(C)}$, где

$$
\mathscr{B}_{P}=\mathscr{B} \otimes L\left(\sqrt[n]{a_{1}}, \ldots, \sqrt[n]{a_{N}}\right)(C)_{P}
$$

а $P$ - некоторая $L\left(\sqrt[n]{a_{1}}, \ldots, \sqrt[n]{a_{N}}\right)$-рациональная точка. Так как $L\left(\sqrt[n]{a_{1}}, \ldots\right.$, $\left.\sqrt[n]{a_{N}}\right)-P_{n, r}$-поле, то $i(\mathscr{B}) \leqslant n^{r+2 g(C)}$. Расширив алгебру $\mathscr{A}$ на расширение степени, менњшей либо равной $t n^{N}=p_{1}^{m_{1}} \cdots p_{q}^{m_{q}} u n^{N}$, получим алгебру индекса, не превосходящего $n^{r+2 g(C)}$. Откуда $i(A) \leqslant n^{r+N+2 g(C)} p_{1}^{m_{1}} \cdots p_{q}^{m_{q}}$.

Второй результат основан на следующей конструкции. Пусть $k-P_{n, r}$-поле, $C$ - плоская проективная кривая над $k$, определенная уравнением $F(x, y)=0$, имеющая $k$-рациональную точку $P$, и пусть $\mathscr{A}$ - ц.п.а. над $K(C)$ экспоненты $n=$ $p_{1}^{n_{1}} \cdots p_{q}^{n_{q}}$. Предположим, что $t=p_{1}^{m_{1}} \cdots p_{q}^{m_{q}} w$, где НОД $(n, w)=1,-$ минимальная из степеней многочлена $F(x, y)$ по переменным $x$ и $y, \mathscr{A}$ разветвлена в замкнутых точках $P_{1}, \ldots, P_{s}$ кривой $C$. Пусть $Q_{1}, \ldots, Q_{m}$ - различные замкнутые точки проективной прямой $\mathbb{P}_{k}^{1}$, лежащие под точками $P_{i}$, и $g$ - произведение всех неприводимых унитарных многочленов, соответствующих конечньм точкам, лежащим под точками $P_{i}$.

Если все точки $Q_{i}$ конечные, положим $u=g(P)^{t n-1}$ в случае $g(P) \neq 0$ и $u=1$ в случае $g(P)=0$. Пусть $H=g u$.

Если одна из точек $Q_{i}$ является бесконечной и НОД $(\operatorname{deg} g, n)=1$, то положим $H=g u$, где $u$ определено как и выше. Если НОД $(\operatorname{deg} g, n) \neq 1$, то пусть $v$ - линейный многочлен такой, что НОД $(v, g)=1$, и $m$ - наименњшее целое положительное число со свойством НОД $(\operatorname{deg} g+m, n)=1$. Положим $H=g v^{m} u$, где

$$
u= \begin{cases}\left(g(P) v^{m}(P)\right)^{t n-1}, & \text { если } g(P) v^{m}(P) \neq 0 ; \\ 1, & \text { если } g(P) v^{m}(P)=0 .\end{cases}
$$

Пусть $C_{1}$ - кривая с полем функций $k\left(C_{1}\right)=k(C)(\sqrt[n t]{H})$. Пусть остаются в силе вьшеприведенные обозначения.

ТЕОРема 17. Справедливо равенство

$$
i(\mathscr{A})=n^{2 g\left(C_{1}\right)+r+1} p_{1}^{m-1} \cdots p_{q}^{m_{q}} .
$$


ДоказАтельство. Алгебра $\mathscr{A} \otimes k\left(C_{1}\right)$ не разветвлена. Кроме того, по построению многочлена $H$ кривая $C_{1}$ обладает $k$-рациональной точкой. Тогда ввиду теоремы ван ден Берга $i\left(\mathscr{A} \otimes k\left(C_{1}\right)\right) \leqslant n^{r+2 g\left(C_{1}\right)}$, что дает $i(\mathscr{A}) \leqslant n^{r+1+2 g\left(C_{1}\right)} \times$ $p_{1}^{m_{1}} \ldots p_{q}^{m_{q}}$. Теорема доказана.

ЗАмечАниЕ. Заметим, что в случае специальных кривых и экспонент оценка (5) является более точной, чем (4).

\section{Список литературы}

1. Albert A. A. Structure of algebras. New York: Amer. Math. Soc., 1939. (Amer. Math. Soc. Colloquium Publ. V. 24.)

2. Deuring $M$. Algebren. Berlin: Springer-Verlag, 1968. (Ergebnisse der Mathematik und ihrer Grenzgebiete. V. 41.)

3. Жеглов А.Б. О строении двумерных локальных тел // Изв. РАН. Сер. матем. 2001. T. 65. № 1. C. $25-60$.

4. Colliot-Thelénèm J.-L., Ojanguren M., Parimala R. Quadratic forms over fraction fields of two-dimensional Henselian rings and Brauer groups of related schemes. Orsay: d'Université de Paris - Sud., 2000-40.

5. Brauer $R$. Untersuchungen über die arithmetischen Eigenschften von Gruppen linearer Substitutionen // Math. Z. 1929. V. 31. P. 733-747.

6. Saltman D. J. Division algebras over $p$-adic curves // J. Ramanujan Math. Soc. 1997. V. 12. № 21. P. 25-47.

7. Saltman D. J. Correction to: Division algebras over $p$-adic curves // J. Ramanujan Math. Soc. 1998. V. 13. № 2. P. 125-129.

8. van den Bergh $M$. Algebraic subfields and splitting fields of division algebras over function fields // Ph.D. thesis. Wilrijk: Universiteit Antwerpen, 1985.

9. Steiner P.A.J. Groupe de Brauer des corps de fractions rationnelles à coefficients complexes // Enseign. Math. (2). 1984. V. 30. № 1-2. P. 115-140.

10. Rehmann U., Tikhonov S.V., Yanchevskii V.I. Two-torsion of the Brauer goups of hyperelliptic curves and unramified algebras over their function fields // Comm. Algebra. 2001. V. 29. № 9. P. 3971-3987.

11. Margolin G.L., Rehmann U., Yanchevskii V.I. Generation of the 2-torsion part of the Brauer group of local quintic by quaternion algebras, the totally splitting case // Algebraic K-theory and its applications (Trieste, 1997). Singapore: World Scientific, 1999. P. 503-535.

12. Танкеев С. Г. О группе Брауэра // Изв. РАН. Сер. матем. 2000. Т. 64. № 4. С. 787-806.

13. Танкеев С. Г. О группе Брауэра арифметической схемы // Изв. РАН. Сер. матем. 2001. T. 65. № 2. C. 155-186.

14. Colliot-Thelénè J.-L., Sansuc J.-J. The rationality problem for fields of invariants under algebraic groups (with special regards to the Brauer group) // Lecture Notes from the 9th ELAM, Santiago de Chile, 1988.

15. Фаддеев Д. К. Простые алгебры над полем алгебраических функций от одной переменной // Труды МИАН. 1951. Т. 38. С. 321-344.

16. Grothendieck A. Le groupe de Brauer // Dix Exposés sur la Cohomologie des Schémas. Amsterdam: North-Holland, 1968. P. 46-188.

17. Colliot-Thélène J.-L., Swinnerton-Dyer P. Hasse principle and weak approximation for pencils of Severi-Brauer and similar varieties // J. Reine Angew. Math. 1994. V. 453. P. 49-112.

18. Bloch $S$. Torsion algebraic cycles, $K_{2}$, and Brauer goups of function fields // Bull. Amer. Math. Soc. 1974. V. 80. P. 941-945. 